\title{
Detection of a Spin Accumulation in Nondegenerate Semiconductors
}

\author{
R. Jansen and B. C. Min \\ $\mathrm{MESA}^{+}$Institute for Nanotechnology, University of Twente, 7500 AE Enschede, The Netherlands
}

(Received 27 July 2007; published 14 December 2007)

\begin{abstract}
Electrical detection of a spin accumulation in a nondegenerate semiconductor using a tunnel barrier and ferromagnetic contact is shown to be fundamentally affected by the energy barrier associated with the depletion region. This prevents the ferromagnet from probing the spin accumulation directly, strongly suppresses the magnetoresistance in current or potentiometric detection, and introduces nonmonotonic variation of spin signals with voltage and temperature. Having no analogue in metallic systems, we identify energy mismatch as an obstacle for spin detection, necessitating control of the energy landscape of spin-tunnel contacts to semiconductors.
\end{abstract}

DOI: 10.1103/PhysRevLett.99.246604

The control and usage of the spin degree of freedom in semiconductor structures lie at the heart of spintronics [14]. Research is fueled by prospects of novel electronic devices (spin transistors and the like) with improved performance or novel functionality and the development of a spin-based quantum computer. Thereby, a wealth of intriguing spin-related phenomena have been discovered in semiconductors [1-3], including carrier-mediated ferromagnetism, long spin-coherence times, control of spin polarization by electric field, strain, or currents, and the spin-Hall effect. Crucial to many spintronic devices is the ability to (i) inject spin-polarized carriers into the semiconductor, (ii) transport them with or without spin manipulation in the semiconductor, and (iii) detect the spins with a second magnetic contact [4-6].

Spin injection and detection has been successful in nonmagnetic metals [7,8], metallic carbon nanotubes [9], and graphene [10]. For semiconductors, progress has been more difficult. A fundamental obstacle identified for spin injection is the conductivity mismatch [11] between a low resistivity ferromagnetic metal (FM) and a semiconductor (SC), causing the injected current to lack significant spin polarization as it is controlled by the large, spinindependent resistance of the SC. This issue was shown to be solved by introducing an extra spin-dependent interface resistance, for instance, a tunnel barrier, between FM and SC $[12,13]$. Despite this solution, significant magnetoresistance in a two-terminal FM/SC/FM device with diffusive transport is yet to be observed. Spin transport in a fully electrical device has been observed in 4-terminal nonlocal geometry (for GaAs [14]) where the resistance of the detection contact is not an issue, or using hot-electron transport (using undoped $\mathrm{Si}[15]$ ) where the conductivity mismatch does not play a role.

Theoretical models [4,11,16-19] for spin transport (and conductivity mismatch) in such SC structures are based on diffusive transport and/or tunneling transport across the FM/SC interface. While this is appropriate for metal systems, it is questionable to describe the $\mathrm{SC}$ as a nonmagnetic metal with low conductivity and flat energy bands as this
PACS numbers: 72.25.Hg, 72.25.Dc, 73.40.Gk, 85.75.-d

neglects the specific energy landscape associated with semiconductor contacts. As this does not capture all the physics, it is important to go beyond this and include band bending, carrier depletion, and thermally activated transport in the SC.

Here we evaluate the general case of a ferromagnetic tunnel contact on a nondegenerate semiconductor. We show that the depletion region crucially affects spin flow across the contact and severely compromises the ability to detect a spin accumulation in the SC. We find that magnetoresistance of such contacts is suppressed and identify energy mismatch as a fundamental obstacle for spin detection not present in metallic structures. The results apply to many nondegenerate semiconductors, including III-V (e.g., GaAs) and group IV ( $\mathrm{Si}, \mathrm{Ge}$ ) materials, organic semiconductors, and semiconducting carbon nanotubes or graphene quantum structures.

Compared to tunneling between two FMs, a fundamental difference arises in a FM/I/SC junction (I a tunnel insulator). The spin polarization of a FM has its origin in the exchange interaction, causing a spin splitting of the density of states (DOS) over a wide energy interval around the Fermi energy $\epsilon_{F}$. The spin-dependent electronic structure of the FM/I interface, together with spin-dependent tunnel matrix elements, produces a nonzero tunnel spin polarization. In contrast, in a SC it is the occupation of the states that is spin dependent. In thermal equilibrium such a spin accumulation is described by a spin-dependent electrochemical potential $\mu^{\sigma}=\mu^{0} \pm \Delta \mu$, where $\sigma$ denotes the spin variable (up $\uparrow$ or down $\downarrow$ ), $\mu^{0}$ is the average electrochemical potential in the SC, and $\Delta \mu$ is half of the spin splitting (all in eV). Hence, one obtains a spindependent Fermi distribution function $f^{\sigma}\left(\epsilon, \mu^{\sigma}\right)=$ $1 /\left(\exp \left[\left(\epsilon-\mu^{\sigma}\right) / \mathrm{kT}\right]+1\right)$, where $\epsilon$ is the energy, $k$ the Boltzmann's constant, and $T$ the temperature. The absolute difference in occupation $f^{\uparrow}-f^{\downarrow}$ is appreciable only in a narrow energy interval around $\mu^{\downarrow}$ and $\mu^{\uparrow}$, and zero elsewhere. However, the spin polarization of the state occupation $\left(f^{\uparrow}-f^{\downarrow}\right) /\left(f^{\uparrow}+f^{\downarrow}\right)$ is zero at energies more than a few kT below $\mu^{\downarrow}$, sharply rises, and for energies more than a 
few kT above $\mu^{\uparrow}$, approaches a constant, nonzero value equal to $\tanh (\Delta \mu / \mathrm{kT})$. This is independent of energy, but sensitive to temperature. For instance, for $\Delta \mu=5 \mathrm{meV}$, the spin polarization of electrons at all energies more than a few $\mathrm{kT}$ above the electrochemical potential in the SC is $19.1 \%$ at $300 \mathrm{~K}, 52.3 \%$ at $100 \mathrm{~K}$, and $100 \%$ at $10 \mathrm{~K}$. Since these electrons are in the high energy tail of the distribution, their contribution to tunneling between metals or through a narrow depletion region is negligible; however, they dominate transport in tunnel junctions with a nondegenerate SC.

We consider a FM/I/SC tunnel junction with an $n$-type SC, separated from the FM by an artificial tunnel barrier of width $w$ and height $\phi$ (Fig. 1). Associated with the FM/I interface is a tunnel spin polarization $\mathrm{P}_{\mathrm{FM}}$. The energy bands in the SC bend upward and define a depletion region that for a nondegenerate $\mathrm{SC}$ is in general so wide that tunneling is negligible and transport is dominated by thermionic emission over the barrier [20]. The spinaveraged Schottky barrier height $\Phi_{B}$ is defined at zero applied voltage as the energy difference between the conduction band minimum at the SC surface (not spin dependent), and the average electrochemical potential $\mu^{0}$ in the bulk of the SC, taken as ground $\left(\mu^{0}=0\right)$. To keep the calculation general, we assume that in the bulk of the SC a constant spin accumulation is maintained, independent of the charge and spin flow across the tunnel detector contact. In practice, this depends on the source of the spin accumulation and on the specific device design parameters. A bias $V_{m}$ (in units of eV) is applied to the FM electrode and we seek the resulting value of the potential $V_{s}^{\sigma}$ at the I/SC interface $(x=0)$ for each spin. This defines $f^{\uparrow}(\epsilon)$ and $f^{\downarrow}(\epsilon)$ at the SC interface and thereby the tunneling current $J_{\text {tun }}$ between this interface and the FM, as well as the thermionic emission current $J_{\text {th }}$ between the SC bulk and SC

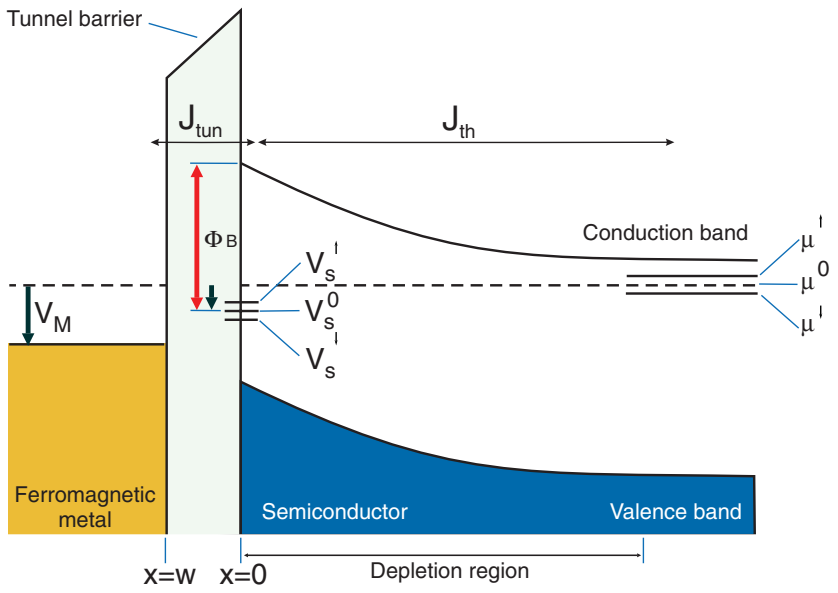

FIG. 1 (color). Energy band diagram of a FM/I/SC tunnel junction with an $n$-type nondegenerate SC having a spin-split electrochemical potential $\mu$ in its bulk. The horizontal axis is not to scale, as $w$ is typically $1 \mathrm{~nm}$, while the depletion region is much wider (few $100 \mathrm{~nm}$ ). surface across the depletion region. For a certain value of $V_{m}$, the $V_{s}^{\sigma}$ values are found self-consistently by requiring $J_{\text {th }}=J_{\text {tun }}$ for each spin separately. This neglects spin relaxation in the SC so as to highlight the effects of the depletion region on spin transport. Including spin relaxation is straightforward and merely reduces spin signals.

For the thermionic emission current $J_{\text {th }}$ we have:

$$
\begin{gathered}
J_{\mathrm{th}}^{\uparrow \downarrow}=J_{0}\left\{\exp \left[\beta\left(V_{s}^{0} \pm \Delta \mu\right)\right]-\exp \left( \pm \beta \Delta V_{s}\right)\right\}, \\
J_{0}=A^{* *} T^{2} \exp \left(-\beta \Phi_{B}\right),
\end{gathered}
$$

where $\beta=1 / \mathrm{kT}, A^{* *}$ the modified Richardson constant [20], and + signs are for $\uparrow$-spin. For the SC surface we defined $V_{s}^{0}=\left(V_{s}^{\uparrow}+V_{s}^{\downarrow}\right) / 2$ and half of the spin splitting $\Delta V_{s}=\left|V_{s}^{\uparrow}-V_{s}^{\downarrow}\right| / 2$. The first term in Eq. (1) corresponds to emission of electrons from the SC bulk to the surface, as governed by the Fermi distribution in the bulk (hence the factor $\pm \Delta \mu$ ) and the (not spin-dependent) energy position of the conduction band minimum at $x=0$, given by $\Phi_{B}-$ $V_{s}^{0}$. The second term in Eq. (1) corresponds to emission of electrons from the SC surface to the bulk that see a barrier height of $\Phi_{B} \mp \Delta V_{s}$. Key is that the first term does not depend on the spin splitting $\Delta V_{s}$ at the SC surface, while the second term does not depend on the spin accumulation $\Delta \mu$ in the SC bulk.

The tunnel current in free electron approximation [21] is given as an integral over energy (longitudinal $\epsilon_{x}$, radial $\epsilon_{r}$ ) restricted to be above the conduction band minimum at the SC surface:

$$
\begin{aligned}
J_{\mathrm{tun}}^{\uparrow, \downarrow}= & \frac{4 \pi m^{2} q}{h^{3}} \int_{\Phi_{B}-V_{s}^{0}}^{\infty} \int_{0}^{\infty}\left(1 \pm P_{\mathrm{FM}}\right)\left[f\left(\epsilon,-V_{s}^{0} \mp \Delta V_{s}\right)\right. \\
& \left.-f\left(\epsilon,-V_{m}\right)\right] T\left(\epsilon_{x}\right) d \epsilon_{x} d \epsilon_{r}
\end{aligned}
$$

with $m$ the electron mass and $h$ Planck's constant. The tunnel transmission factor $T\left(\epsilon_{x}\right)$ is

$$
\exp \left\{\frac{-4 \pi}{h} \int_{0}^{w} \sqrt{2 m\left[\phi-\Phi_{B}-\left(V_{m}-V_{s}^{0}\right)(x / w)-\epsilon_{x}\right]} d x\right\} \text {. }
$$

In Eq. (3), the spin polarization of the SC surface enters via the Fermi distribution. The tunnel spin polarization of the FM was taken to be $30 \%$ at $\boldsymbol{\epsilon}_{F}$ and decays with energy as $\mathrm{P}_{\mathrm{FM}}=0.3 \exp \left(-\left|\epsilon-\epsilon_{F}\right| / 0.55\right)$, mimicking observations for $\mathrm{FM} / \mathrm{Al}_{2} \mathrm{O}_{3}$ interfaces [22,23]. Below we present results for $n$-type $\mathrm{Si}\left(A^{* *}=110 \mathrm{~A} \mathrm{~K}^{-2} \mathrm{~cm}^{-2}\right)$ and a tunnel barrier with $w=1 \mathrm{~nm}, \phi=1.5 \mathrm{eV}$.

Figure 2, top panel, shows the calculated values of $\Delta V_{s}$ versus applied bias $V_{m}$ for orientation of the magnetization of the FM either parallel (P) or antiparallel (AP) to the spin accumulation in the SC bulk (for $\Phi_{B}=600 \mathrm{meV}$ ). We observe that for negative bias (electrons from FM into $\mathrm{SC})$ the spin accumulation at the SC surface vanishes, even though $\Delta \mu$ was fixed at $5 \mathrm{meV}$. In this regime the Schottky barrier is in reverse bias and the small thermionic 


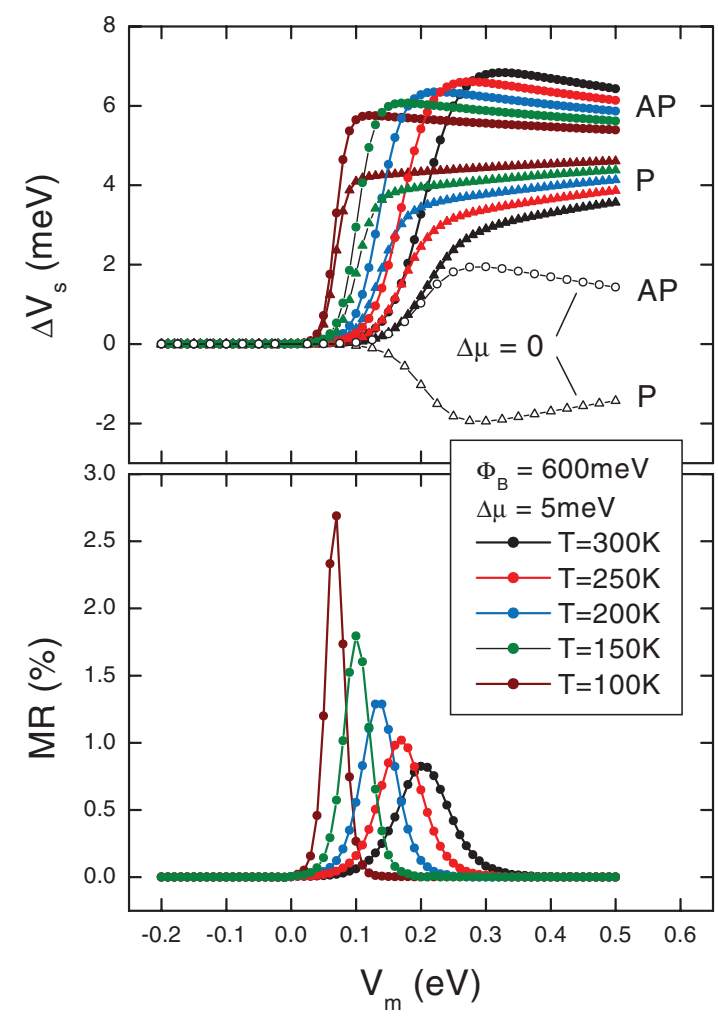

FIG. 2 (color). Top panel: calculated spin splitting $\Delta V_{s}$ at the semiconductor surface for P (triangles) and AP (circles) state, as a function of $V_{m}$, for different values of $T$ as indicated. The spin accumulation $\Delta \mu$ in the bulk of the semiconductor is $5 \mathrm{meV}$ (solid color symbols) or $0 \mathrm{meV}$ (open symbols). Bottom panel: resulting magnetoresistance.

emission current [second term in Eq. (1)] limits the flow across the contact. All voltage drops over the SC depletion region and $V_{s}^{\sigma} \approx V_{m}$ for both spins. Hence the magnetoresistance $\left[\mathrm{MR}=\left(J^{\mathrm{P}}-J^{\mathrm{AP}}\right) / J^{\mathrm{AP}}\right]$ vanishes (bottom panel). This resembles the conductivity mismatch [11], although the mismatch here is due to the large resistance associated with the depletion region.

A nonzero spin accumulation $\Delta V_{s}$ at the SC surface exists only for large enough positive bias. With increasing forward bias the effective barrier for thermionic emission from the SC bulk to the surface decreases. This exponentially decreases the resistance of the depletion region, until the flow across the contact becomes limited by the tunneling step across the insulator. As can be seen in Fig. 2, top panel, $\Delta V_{s}$ now also depends on the magnetization alignment (P or AP). Note that $\Delta V_{s}^{\mathrm{AP}}>\Delta \mu$ while $\Delta V_{s}^{\mathrm{P}}<\Delta \mu$, and that their difference is reduced at larger $V_{m}$, which is due to the decay of $\mathrm{P}_{\mathrm{FM}}$ with energy. Despite the nonzero $\Delta V_{s}$, remarkably, the MR still vanishes except for a narrow interval corresponding to the transition region (bottom panel). For large positive $V_{m}$ the MR is zero because the FM electrode is decoupled from the spin accumulation in the SC bulk due to the depletion region. When the magnetization of the FM and thus $\mathrm{P}_{\mathrm{FM}}$ is reversed, $J_{\text {tun }}$ for each spin changes. Since $J_{\text {tun }}$ depends on $V_{s}^{\uparrow}$ and $V_{s}^{\downarrow}$ [Eq. (3)], these will adjust to reach a new steady state with $J_{\text {th }}=J_{\text {tun }}$. However, since $J_{\text {th }}$ in this bias regime is governed by the first term in Eq. (1) that does not depend on $\Delta V_{s}$ the total current does not change. Hence MR $=0$. For similar reasons, the spin polarization of the current, while nonzero, is independent of the magnetization of the FM (P or AP). The first term of Eq. (1) dominates and the thermionic emission has a spin polarization that is easily obtained as $\tanh (\Delta \mu / \mathrm{kT})$. This is equal to that of the electrons in the tail of the Fermi distribution in the SC bulk and independent of $V_{s}^{\dagger}, V_{s}^{\downarrow}$, and $\mathrm{P}_{\mathrm{FM}}$.

Note that for $\Delta \mu=0$, the spin polarization of the current vanishes $[\tanh (\Delta \mu / \mathrm{kT})=0]$, but $\Delta V_{s}$ does not, and it has a sign that depends on the magnetization direction of the FM. Thus, extraction of electrons from the SC by a FM tunnel contact leads to a significant surface spin accumulation of several $\mathrm{meV}$, as determined by $\mathrm{P}_{\mathrm{FM}}$. A final yet important observation is that the calculated magnitude of the MR varies significantly with temperature, even though all the parameters (including $\Delta \mu, \mathrm{P}_{\mathrm{FM}}$ ) were taken to be constant. In experiment, this may be misinterpreted as being due to changes in $\Delta \mu$ or the spin relaxation with $T$. The predicted nonmonotonic variation of the spin signals with both $V_{m}$ and $T$ can, however, be verified experimentally.

Figure 3 shows results for a $\Delta \mu=5 \mathrm{meV}$, and $\Phi_{B}$ varied from 200 to $800 \mathrm{meV}$. A nonzero MR is obtained

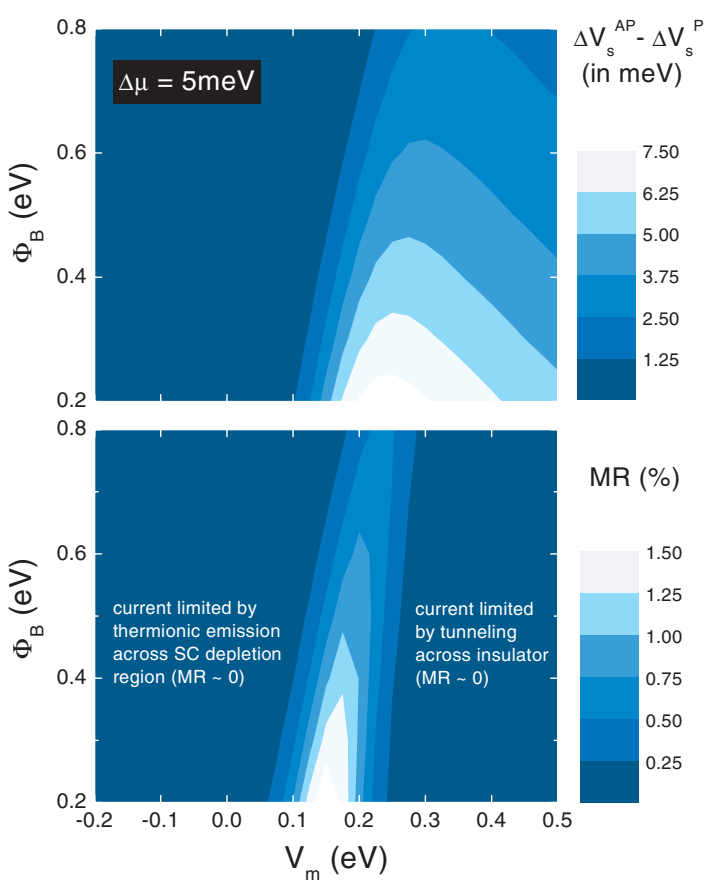

FIG. 3 (color). Top panel: change in the surface spin splitting $\Delta V_{s}$ between $\mathrm{AP}$ and $\mathrm{P}$ state vs $\Phi_{B}$ and $V_{m}$. Bottom panel: resulting magnetoresistance. Calculations for $\Delta \mu=5 \mathrm{meV}$ and $T=300 \mathrm{~K}$. 


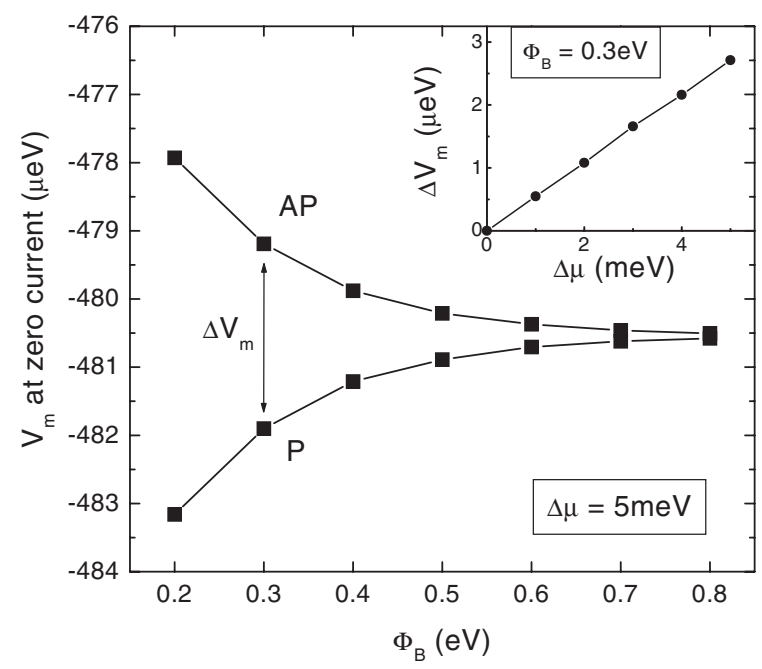

FIG. 4. Bias voltage $V_{m}$ at which the current is zero for AP and P state vs $\Phi_{B}$, for $T=300 \mathrm{~K}$ and $\Delta \mu=5 \mathrm{meV}$. The inset shows $\Delta V_{m}$ vs $\Delta \mu$ for $\Phi_{B}=0.3 \mathrm{eV}$ and $300 \mathrm{~K}$.

in a narrow bias interval, in which the resistance of the tunnel barrier and the depletion region are comparable. This allows the FM to communicate with the spin accumulation in the SC bulk, albeit indirect as transport occurs at a minimum energy defined by $\Phi_{B}$, whereas appreciable differences in the state occupation are confined to energies close the electrochemical potential. As a result, the MR values are rather small $(<1.5 \%)$, notwithstanding the significant tunnel spin polarization and spin accumulation used. Figure 3 also reveals that the maximum MR is reduced with increasing $\Phi_{B}$, which is due to the decay of the tunnel spin polarization $\mathrm{P}_{\mathrm{FM}}$ with energy.

Having established that the depletion region in a FM/I/ SC contact severely hampers detection of a spin accumulation, we examine the nonlocal detection geometry originally developed for metals $[7,8]$. This uses two contacts for injection of a spin accumulation, and two other contacts, one placed within a spin-diffusion length from the injection contact, as voltage probes to detect the spin accumulation. For FM/I/SC contacts with the same parameters as used before, Fig. 4 shows the calculated $V_{m}(J=0)$ sensed in the condition of zero current through the contact (potentiometric detection). Despite the fact that $\Delta \mu=5 \mathrm{meV}$, the detected $V_{m}$ changes only by a few $\mu \mathrm{eV}$ when the magnetization of the FM is changed from $\mathrm{P}$ to $\mathrm{AP}$ orientation. Moreover, the signal decreases rapidly at larger $\Phi_{B}$. Thus, we find that a FM/I/SC junction with nondegenerate SC also renders potentiometric spin detection inefficient. The change in $V_{m}$ from $\mathrm{P}$ to AP is linear in $\Delta \mu$ (see the inset); however, the signal varies with $T$ in a nonmonotonic fashion (not shown) even if all parameters (including $\Delta \mu, \mathrm{P}_{\mathrm{FM}}$ ) are taken to be constant, as also noted for the MR (Fig. 2).

The results show that the depletion region of a nondegenerate SC presents a fundamental obstacle for spin transport in FM-SC devices. The problem is universal and exists for any SC (organic or inorganic, $n$-type or $p$-type) where a depletion region develops that is impenetrable by tunneling, such that the energy at which spin transport occurs is offset from the typical energy scale of the spin accumulation. Approaches to remove the depletion region, such as recently developed for spin-tunnel contacts to $n$-type $\mathrm{Si}$ using ferromagnets with a low work function [24,25], are therefore crucial.

We acknowledge financial support from the NWO-VIDI program and from the Netherlands Nanotechnology Networks NANOIMPULS and NANONED (supported by the Ministry of Economic Affairs).

[1] S. A. Wolf et al., Science 294, 1488 (2001).

[2] I. Zutić, J. Fabian, and S. Das Sarma, Rev. Mod. Phys. 76, 323 (2004).

[3] D. D. Awschalom and M.E. Flatté, Nature Phys. 3, 153 (2007).

[4] A. Fert, J.-M. George, H. Jaffrès, and R. Mattana, IEEE Trans. Electron Devices 54, 921 (2007).

[5] I. Zutić, J. Fabian, and S. C. Erwin, Phys. Rev. Lett. 97, 026602 (2006).

[6] H. Dery, P. Dalal, L. Cywiński, and L. J. Sham, Nature (London) 447, 573 (2007).

[7] M. Johnson and R. H. Silsbee, Phys. Rev. Lett. 55, 1790 (1985).

[8] F. J. Jedema, A. T. Filip, and B. J. van Wees, Nature (London) 410, 345 (2001).

[9] L. E. Hueso et al., Nature (London) 445, 410 (2007).

[10] N. Tombros, C. Jozsa, M. Popinciuc, H. T. Jonkman, and B. J. van Wees, Nature (London) 448, 571 (2007).

[11] G. Schmidt et al., Phys. Rev. B 62, R4790 (2000).

[12] A. T. Hanbicki et al., Appl. Phys. Lett. 80, 1240 (2002); B. T. Jonker, Proc. IEEE 91, 727 (2003).

[13] V.F. Motsnyi et al., Appl. Phys. Lett. 81, 265 (2002); W. Van Roy et al., IEEE Trans. Electron Devices 54, 933 (2007).

[14] X. Lou et al., Nature Phys. 3, 197 (2007).

[15] I. Appelbaum, B. Huang, and D. J. Monsma, Nature (London) 447, 295 (2007).

[16] A. Fert and H. Jaffrès, Phys. Rev. B 64, 184420 (2001).

[17] Z. G. Yu and M. E. Flatté, Phys. Rev. B 66, 201202 (2002).

[18] G. E. W. Bauer et al., Phys. Rev. B 72, 155304 (2005).

[19] H. Dery and L. J. Sham, Phys. Rev. Lett. 98, 046602 (2007).

[20] S.M. Sze, Physics of Semiconductor Devices (Wiley, New York, 1981).

[21] E. L. Wolf, Principles of Electron Tunneling Spectroscopy (Oxford University Press, New York, 1989).

[22] S. O. Valenzuela et al., Phys. Rev. Lett. 94, 196601 (2005).

[23] B. G. Park, T. Banerjee, J. C. Lodder, and R. Jansen Phys. Rev. Lett. 99, 217206 (2007).

[24] B. C. Min, K. Motohashi, J. C. Lodder, and R. Jansen, Nature Mater. 5, 817 (2006).

[25] K. Sugiura, R. Nakane, S. Sugahara, and M. Tanaka, Appl. Phys. Lett. 89, 072110 (2006). 